\title{
Oocysticidal Effect of Essential Oil Components against Chicken Eimeria Oocysts
}

\author{
Adnane Remmal, Sanaa Achahbar, Latifa Bouddine, \\ Fouzia Chami and Najat Chami
}

Laboratory of Biotechnology, Faculty of Sciences Dhar El Mehrez, Fez, Morocco

Correspondence should be addressed to: Adnane Remmal; adnaneremmal@gmail.com

Received 22 January 2013; Accepted 5 February 2013; Published 28 May 2013

Academic Editor: João Luís Garcia

Copyright (C) 2013 Adnane Remmal, Sanaa Achahbar, Latifa Bouddine, Fouzia Chami and Najat Chami. Distributed under Creative Commons CC-BY 3.0

\begin{abstract}
The objective of this study is to assess the ability of some major components of essential oils (EOs) to destroy Eimeria oocysts in vitro and to find out how the mechanism of action involved in this oocysticidal effect works.

The methodology used was as follows: A screening of the oocysticidal activity of eight EO components was carried out in a liquid medium. The release of substances absorbing at $273 \mathrm{~nm}$ was measured after treatment of Eimeria oocysts with these components. The results we obtained showed that carvacrol, carvone, isopulegol, thymol, and eugenol have the most effective activity. The treatment of Eimeria oocysts with these components led to their lysis in a dose and time dependant manner as shown by the release of substances absorbing at $273 \mathrm{~nm}$.

We were able to conclude that these major components are of particular interest in fighting coccidiosis since they have a destructive effect on oocysts at very low concentrations. They could also help in the formulation of radical and safe solutions to coccidiosis.
\end{abstract}

Keywords: Essential oil component, Eimeria oocyst, oocysticidal activity.

\section{Introduction}

Coccidiosis is one of the most common chicken diseases caused by protozoan parasites of the genus Eimeria that can seriously affect the health and the productivity of livestock. Eimeria infection is transmitted through hardy, thick-walled spore oocysts able to survive for long periods in the poultry litter and soil particles. Outside of the animal's body (soil and litter), farmers usually fight this disease by disinfectants against oocysts and treat the animals themselves by antiparasitic drugs against oocysts that have infected the gut of the animal. Environmental oocysts are refractory to most commonly used disinfectants. The only compounds that have been found to consistently have useful oocysticidal activity are ammonia, methyl bromide, carbon disulfide and some phenolic products, as reported by Hilbrish et al. (1975) and Williams et al (1997). On the one hand the toxicity of these products makes them impossible to use for disinfecting the broiler house in presence of animals, and on the other hand, they

Cite this Article as: Adnane Remmal, Sanaa Achahbar, Latifa Bouddine, Fouzia Chami and Najat Chami (2013), "Oocysticidal Effect of Essential Oil Components against Chicken Eimeria Oocysts," International Journal of Veterinary Medicine: Research \& Reports, Vol. 2013 (2013), Article ID 599816, DOI: $10.5171 / 2013.599816$ 
represent a danger to the staff that performs the disinfection. In a research by Chapman (1984), Harper and Makatouni (2002), the problem of resistance to antiparasitic agents used against infecting oocysts has led to higher treatment doses which yielded residues in poultry products. The works published by Giannenas et al (2003), Oviedo-Rondo'n et al (2006) and Da Silva et al (2009) have reported the in vivo efficiency of natural plant extracts in the treatment of coccidiosis.

The works conducted by Rhayour et al (2003), Chami et al $(2004,2005)$ and Bennis et al (2004) have already demonstrated the antimicrobial action of several essential oils (EO) of aromatic plants and some of their components on bacteria and fungi in vitro and in vivo. Recently, Remmal et al (2011) have demonstrated that EOs are efficient to destroy Eimeria oocysts in vitro. Since each EO is a mixture of many components, we tested the oocysticidal activity of some of their major components; in order to verify if they are among the active principles that are responsible of the EO's effect.

\section{Material and Methods}

\section{Eimeria Oocysts Isolation and Purification}

Oocysts used in this work were isolated from fresh feces of broilers suffering from coccidiosis in Morocco. They were purified by flotation in a saturated solution as described in the book by Shirley (1995) and maintained by periodic passage through young chicken in our laboratory. The washed oocysts were then stored at $-20^{\circ} \mathrm{C}$ with $50 \%(\mathrm{v} / \mathrm{v})$ glycerol. We obtained therefore a stock of oocyst from which we drew aliquots for each of the experiments.

\section{The Counting of Oocysts}

The number of oocysts was determined by transferring $25 \mu \mathrm{l}$ of the sample suspension of Eimeria oocysts to Malassez chamber for microscopic examination and counting. Eimeria sp oocysts were counted in 10 fields of view by using standard techniques described by Ryley et al (1976), and the mean number of oocysts per milliliter of sample was calculated. The identification of Eimeria species in the faecal samples used in this work was done on the basis of the microscopic observation allowing the distinction of the Emeiria species according to the method described in the book by Brugere-Picoux and Silim (1992). The percentage of each species in the mixed suspension was approximately $45 \% E$. tenella, 32\% E. maxima, 10\% E. acervulina , $6 \%$ E. necatrix and $7 \%$ E. mitis.

\section{The Effect of EO Components on the Number of Oocysts}

All components used in this study were purchased from Sigma-Aldrich (France). The screening of the ability of the eight components to destroy Eimeria oocysts was carried out. The components were: isopulegol, carvacrol, carvone, eugenol, cineol, cinnamaldehyde, carveol and thymol. They were dispersed in liquid medium containing $0.2 \%$ agar in pure water. This dispersion method has been improved by Remmal et al (1993a and 1993b). Each component was tested with increasing concentrations $(0 ; 0.3 ; 0.5 ; 1 ; 2$; 4; 10 and $20 \mathrm{mg} / \mathrm{mL}$ ). The activity of each component was determined in triplicate in 96 well microplates by incubation at ambient temperature for $24 \mathrm{~h}$ of an inoculum of $20 \mu \mathrm{l}$ containing $1.5210^{7}$ oocysts / $\mathrm{mL}$ put in direct contact with 40 $\mu \mathrm{l}$ of the EO component at various concentrations for a final volume of $200 \mu \mathrm{l}$. We plugged our data on different curves expressing the number of oocysts with respect to the concentration of EO components. The $\mathrm{LC}_{50}$ values was then inferred from these curves.

\section{The Effect of Salinomycin and Robenidine on the Decrease of the Number of Oocysts}

The action of salinomycin and robenidine was tested using the previously described method with EO components with the following concentrations 10, 25, 50 and $100 \mathrm{mg} / \mathrm{mL}$. 
The Decrease of the Oocyst's Number in Parallel with the Release of Substances Absorbing at $273 \mathrm{Nm}$ after Treatment with Increasing Concentrations of EO Components

The release of cellular material absorbing at $273 \mathrm{~nm}$ from oocysts cells treated with increasing concentrations of carvacrol, carvone, isopulegol, thymol, and eugenol was determined. This experiment was performed on aliquots incubated for $24 \mathrm{~h}$ at ambient temperature with one milliliter suspension containing:

- $100 \mu \mathrm{l}$ of washed suspension of Eimeria oocysts at $1.52 \times 10^{7}$ oocysts $/ \mathrm{mL}$.

- $700 \mu \mathrm{l}$ of PBS Phosphate Buffer Saline (PBS) (containing $8 \mathrm{~g} / \mathrm{L} \mathrm{NaCl} ; 0.2 \mathrm{~g} / \mathrm{L}$ $\mathrm{KCl} ; 1.13 \mathrm{~g} / \mathrm{L} \mathrm{Na}_{2} \mathrm{HPO}_{4} ; 2 \mathrm{H}_{2} \mathrm{O}$ and $0.2 \mathrm{~g} / \mathrm{L}$ $\mathrm{KH}_{2} \mathrm{PO}_{4}$ ).

- $200 \mu \mathrm{l}$ increasing concentrations of the selected EO components $(0 ; 0.3 ; 0.5 ; 1 ; 2$; 4; 10 and $20 \mathrm{mg} / \mathrm{mL}$ ).

After incubation, the samples were centrifuged at $320 \mathrm{~g}$ for five minutes at $4^{\circ} \mathrm{C}$. $500 \mu \mathrm{l}$ of the supernatant were used to measure the UV absorption by Beckman spectrophotometer. Preliminary experiment showed that $273 \mathrm{~nm}$ is the absorbance peak of the supernatant regardless of what major component was used. Correction was made for the absorption of the suspending liquid containing the same concentration of the component centrifuged after two minutes of contact with oocysts. For each concentration, the oocyst number was counted as previously described.

Time Course of the Decrease of the Oocyst Number, in Parallel with the Release of Substances Absorbing at $273 \mathrm{Nm}$ after Treatment with $4 \mathrm{Mg} / \mathrm{MI}$ of the EO Component

$100 \mu \mathrm{L}$ of washed suspension oocysts incubated with $200 \mu \mathrm{l}$ components at $4 \mathrm{mg} / \mathrm{mL}$ and $700 \mu \mathrm{L}$ of PBS at ambient temperature were treated for different periods of time: $0 \mathrm{~min}, 30 \mathrm{~min}, 1 ; 2 ; 3 ; 4 ; 5$ and $24 \mathrm{~h}$. After incubation, the samples were centrifuged at $320 \mathrm{~g}$ for five minutes at $4^{\circ} \mathrm{C} .500 \mu \mathrm{l}$ of the supernatant were used to measure the UV absorption by Beckman spectrophotometer. After each period of time, the oocyst number was counted as previously described. All tests have been repeated three times under the same conditions.

\section{Results}

The screening of the eight EO components tested shows that the number of oocyst decreases after the treatment with the majority of the components used in a dose dependent manner at a concentration ranging between 0.3 and $20 \mathrm{mg} / \mathrm{ml}$. When expressed in terms of lethal concentration $50 \%\left(\mathrm{LC}_{50}\right)$ (Table 1$)$, our results show that the most efficacious components are carvacrol followed by carvone, isopulegol, thymol, and eugenol according to their $\mathrm{LC}_{50}$ less than $2 \mathrm{mg} / \mathrm{ml}$.

Table 1: LC 50\% of Eight EO Components

\begin{tabular}{|l|l|}
\hline Components & $\mathrm{LC}_{50}(\mathrm{mg} / \mathrm{mL})$ \\
\hline Isopulegol & $1.66 \pm 0.22$ \\
\hline Thymol & $1.66 \pm 0.44$ \\
\hline Eugenol & $1.83 \pm 0.22$ \\
\hline Carvone & $1.54 \pm 0.33$ \\
\hline Carvacrol & $1.50 \pm 0.33$ \\
\hline Cineol & $2.33 \pm 0.44$ \\
\hline carveol & $3.33 \pm 0.44$ \\
\hline Cinnamaldehyde & $3.66 \pm 0.44$ \\
\hline
\end{tabular}

\section{The Effect of Salinomycin and Robenidine}

A dose-response test shows that the number of oocyst decreased slightly with the increasing concentrations of the two antiparasitic drugs tested at the concentrations $10,25,50$ and $100 \mathrm{mg} / \mathrm{mL}$. The salinomycin effect is more pronounced 
than the robenidine one (result not shown).

The Effect of EO Components Concentrations on Eimeria Oocyst Number and the Release of $273 \mathrm{Nm}$ Absorbing Material after $24 \mathrm{H}$ Treatment

Figures $1 \mathrm{~A}, 1 \mathrm{~B}, 1 \mathrm{C}, 1 \mathrm{D}$ and $1 \mathrm{E}$ show that after adding the EO components of carvacrol, carvone, isopulegol, thymol or eugenol in concentrations ranging from 0.3 to $20 \mathrm{mg} / \mathrm{ml}$, the number of oocysts noticeably decreases with very low concentrations ( 0.3 to $2 \mathrm{mg} / \mathrm{ml}$ ). This treatment causes a considerable release of $273 \mathrm{~nm}$ absorbing material that increases in a linear manner according to the concentration of the oils.

A
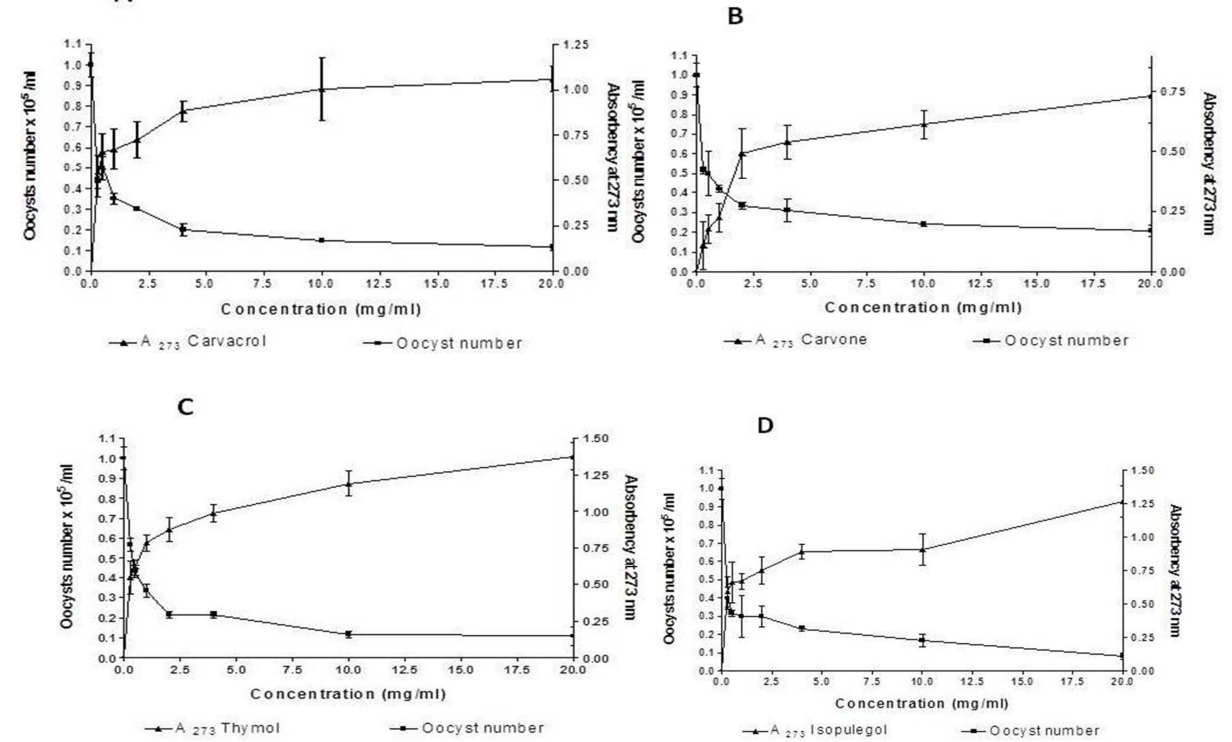

- A $_{273}$ Carvone

- oocyst number

D

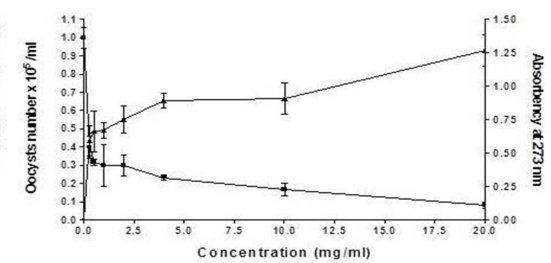

$\rightarrow$ a 2 rs Isopulegol $\rightarrow$ Oocyst number

E

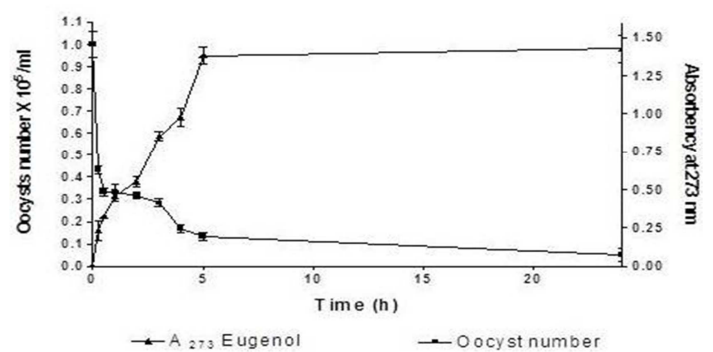

Figure 1: The Effect of Carvacrol (A), Carvone (B), Thymol (C), Isopulegol (D), and Eugenol (E) Concentrations on Eimeria Oocysts Number and the Release of 273nm Absorbing Material

Time Course of the Decrease of the Oocysts Number and $273 \mathrm{Nm}$ Absorbing Material Release from Eimeria Oocyst Treated by $4 \mathrm{mg} / \mathrm{MI}$ of EO Components

Figures 2A, 2B, 2C, 2D and 2E show that after treatment with $4 \mathrm{mg} / \mathrm{ml}$ of carvacrol, carvone, isopulegol, thymol or eugenol for various periods of time, four hours contact were enough to reduce approximately $90 \%$ of the oocyst number. While this decrease of the number of oocysts occurs, we notice an increase of the release of $273 \mathrm{~nm}$ absorbing material from Emeiria oocyst that reached a maximum value approximately after five hours. 
A

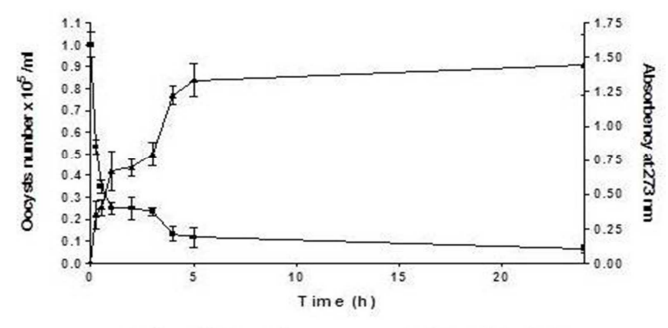

C

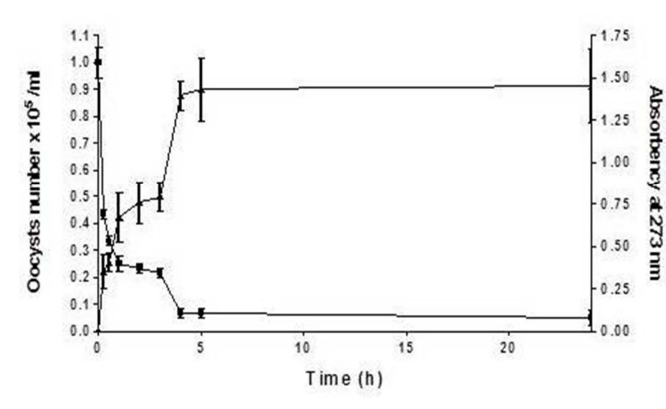

$\rightarrow A_{273}$ Thymol $\rightarrow$ Oocyst number

$\mathrm{E}$
B

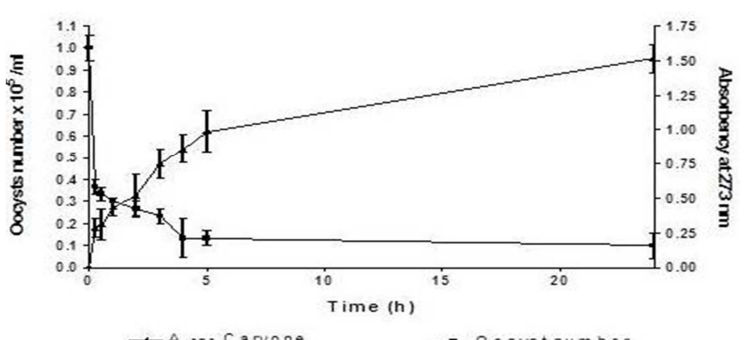

D

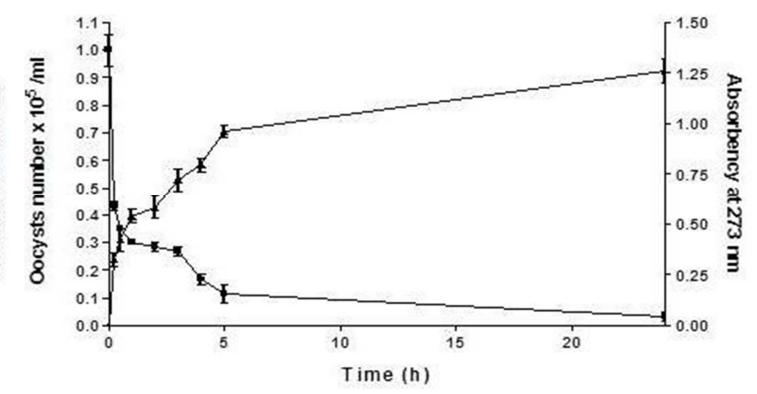

$\rightarrow$ A 273 lsopulegol $\rightarrow$ Oocyst number

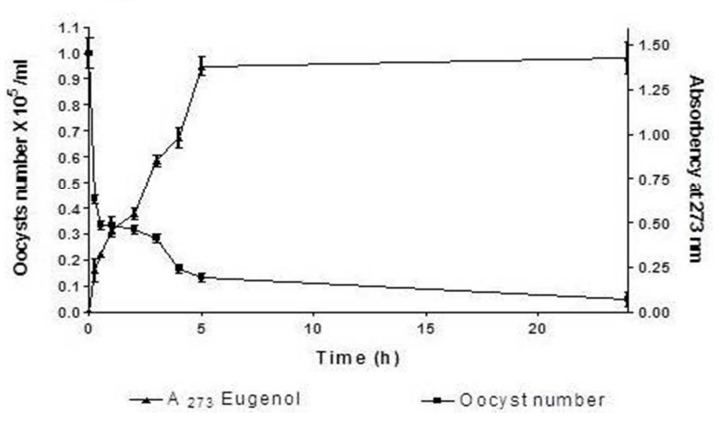

Figure 2: Time Course of the Decrease of the Oocysts Number and 273nm Absorbing Material Release from Eimeria Oocysts Treated by $4 \mathrm{Mg} / \mathrm{Ml}$ of Carvacrol (A), Carvone (B), Thymol (C), Isopulegol (D), and Eugenol (E)

\section{Discussion}

The control of coccidiosis using disinfectant agents in broiler house and the anticoccidial drugs administrated to chicken raise several issues that can be summarized in the following: Safety of staff who perform the disinfection, inability to use disinfectants in presence of animals because of their toxicity, the growing resistance to anticoccidial drugs mentioned by Stephan et al (1997) and the presence of residues of the anticoccidial drugs in poultry products already reported by McEvoy (2002) . These predicaments pushed us to look for a non-toxic alternative that has oocysticidal activity in vitro and that we could use as a disinfectant even in the presence of animals and as preventive or curative therapeutics. In addition to that, this treatment should not be of any threat to staff, animals and consumer.

Essential oils have been demonstrated to have oocysticidal activity in vitro as described by Remmal et al (2011). This is supported by many in vivo studies published by Greathead and Kamel (2006) and Da Silva et al (2009) that reported 
positive results after the treatment of experimental coccidiosis by some EOs or by thymol or carvacrol. In this work we are interested in testing the in vitro oocysticidal effect of some EO components and studying their mechanism of action.

The in vitro test was carried out in aqueous medium without detergent or solvent, using $0.2 \%$ agar as a dispersing agent of EO components according to the method developed by Remmal et al (1993a and 1993b). The release of substances absorbing UV was the evidence used to determine the oocysticidal mechanism of action. This method was used by Dixon and Chopra (1986) and Rhayour et al (2003) to demonstrate the bactericidal action of polymixine $\mathrm{B}$. The fungicidal action of EO component has also been demonstrated by the same method by Bennis et al (2004).

We started the study by carrying out a screening in which we tested eight EO's major components susceptible to damage oocysts because of their phenolic or aldehyde nature that have already been cited in published tests in vivo. The anticoccidial activity of thymol and carvacrol, which are the main constituents of the oregano and thyme EOs have been reported in in vivo tests by Ibrir et al (2001). The cineol and isopulegol along with the cinnamaldehyde and eugenol, carvone and carveol are used as poultry food additives in a research by Lee et al (2003 and 2004). Our results show that five phenolic components among the eight tested (carvacrol, carvone, isopulegol, thymol and eugenol), provoke an oocysticidal effect at low doses with an $\mathrm{LC}_{50}$ less than $2 \mathrm{mg} / \mathrm{ml}$. These results confirm the published data in vivo showing positive action of EOs containing phenolic compounds in the treatment of the experimental coccidiosis cited above. They are also consistent with data showing the action of some oocysticidal phenolic chemical detergents in a research by Williams (1997).

In parallel with the screening carried out on the eight components of EOs, we have tested the ability of salinomycin and robenidine to destroy Eimeria oocyst.
These two anticoccidial agents, normally used as food additives showed that after treatment with increasing concentrations, the oocyst's number slightly decreased, while in the same conditions, the EOs components reduced the number of oocysts in a much more significant proportion. This result confirms the coccidiostatic known effect of the two drugs reported by Wilson (1976) and Schäfer et al (1984) and reveals the oocysticidal effect of EOs' components tested.

During the microscopic counting of the oocysts treated by EO components, we have noted the presence of deformed oocysts with cracked wall and debris (results not shown). Hence the idea to shed light on the mechanism of action by evaluating the lysis of oocysts treated with a range of concentrations of the five powerful EO components selected. The oocyst lysis was determined by measuring the release of substances absorbing at 273 $\mathrm{nm}$ after treatment. The UV-absorbing material is an indication of the release of intracellular contents such as aromatic amino acids and nucleotides. The obtained results showed a parallelism between the decrease of the oocyst number and the increase of the $273 \mathrm{~nm}$ absorbing material in a dose-dependent manner after treatment with EO components. This allows us to conclude that the decrease in the oocysts number is due to an oocysticidal action of the tested molecules. To determine the time needed to observe this oocysticidal action, we have done a time course of the oocyst number reduction and oocyst lysis using the concentration $4 \mathrm{mg} / \mathrm{ml}$. This concentration was chosen because it induces approximately 85 to $90 \%$ reduction oocyst number. We found that four hours contact were sufficient to destroy the majority of oocysts. Preliminary experiments have shown that no spontaneous lysis occurred with untreated oocysts after 24 hours in our experimental conditions. These in vitro results explain the positive effects obtained in vivo by Ibrir et al (2001) with thymol and carvacrol. A study conducted in our laboratory on the in vivo action of these five EOs' components on experimentally 
infected chicken yielded preliminary results that confirm their coccicidal action without resistance or recurrence after treatment interruption (results not shown).

From this in vitro study, it can be concluded that carvacrol, thymol, isopulegol, eugenol and carvone should be of particular interest since they have a destructive effect on oocysts at very low concentrations. To our knowledge, this is the first time that carvone, eugenol and isopulegol are tested in the context of the fight against coccidiosis. This work shows that the major components of essential oils tested separately possess an oocysticidal activity equivalent to that of the whole essential oils which are complex mixtures of ingredients. In our opinion, the use of these molecules in a pure state provides a better pharmacological and toxicological safety.

\section{Acknowledgements}

The authors are deeply grateful to Prof, Mohammed Bennis from the department of English in the faculty of letters, Dhar El Mehrez Fez and to Miss Imane Remmal from Al Akhawayn University for their assistance in checking the English of the manuscript.

\section{References}

Bennis, S., Chami, N., Chami, F., Bouchikhi, T. \& Remmal, A. (2004). "Surface Alteration of Saccharomyces Cerevisiae Induced by Thymol and Eugenol," Letters in Applied Microbiology; 38 454-458.

Chami, F., Chami, N., Bennis, S., Bouchikhi, T. \& Remmal, A. (2005). "Oregano and Clove Essential Oils Induce Surface Alteration of Saccharomyces Cerevisiae," Phytotherapy Research, 19 (5) 405-408.

Chami, F., Chami, N., Bennis, S., Trouillas, J. \& Remmal, A. (2004). "Evaluation of Carvacrol and Eugenol as Prophylaxis and Treatment of Vaginal Candidiasis in an Immunosuppressed Rat Model," Journal of Antimicrobial Chemotherapy, 54 909-14.
Chapman, H. D. (1984). "Drug Resistance in Avian Coccidia (A Review)," Veterinary Parasitology, 15 11- 27.

Dasilva, M. A, Pessotti, B. M. S., Zanini, S. F., Colnago, G. L. Rodrigues, M. R. A., Nunes, L. C., Zanini, M. S. \& Martins, I. V. F. (2009). "Intestinal Mucosa Structure of Broiler Chickens Infected Experimentally with Eimeria Tenella and Treated with Essential Oil of Oregano," Ciência Rural, Santa Maria, 39 (5) 1471-1477.

Dixon, R. A. \& Chopra, I. (1986). "Polymyxin $B$ and Polymyxin B Nonapeptide Alter Cytoplasmic Membrane Permeability in Escherichia Coli," Journal of Antimicrobial Chemotherapy, 18 557-63.

Giannenas, I., Florou-Paneri, P., Papazahariadou, M., Christaki, E., Botsoglou, N. A. \& Spais, A. B. (2003). "Effect of Dietary Supplementation with Oregano Essential Oil on Performance of Broilers after Experimental Infection with Eimeria Tenella," Archiv Für Tierernährung; 57: 99-106.

Greathead, H. \& Kamel, C. (2006). 'Encapsulated Plant Extracts to Fight Coccidiosis,' Feed Mix, 14 18-21.

Guimaraes Junior, J. S., Bogado, A. L. G., Da Cunha, T. C. B. \& Garcia, J. L. (2007). "In Vitro Evaluation of the Disinfection Efficacy on Eimeria Tenella Unsporulated Oocysts Isolated from Broilers," Brazilian Journal of Veterinary Parasitology, 16 67-71.

Harper, C. G. \& Makatouni, A. (2002). "Consumer Perception of Organic Food Production and Farm Animal Welfare," Brazilian Food Journal, 104 287-299.

Hilbrich, P. (1975). 'Disinfection Tests on Eimeria Tenella Oocysts,' Berliner Und Munchener Tierarztliche Wochenschrift; 88 144-8. 
Ibrir, F., Greathead, H. M. R. \& Forbes, J. M. (2001). The Effect of Thymol/Carvacrol Treatments on the Performance of Broiler Chickens Infected with Eimeria Acervulina. Procedure Alternative Feed Antibiotics Anticoccidials in the Pig and Poultry. Meat Production. Oslo.

Lee, K. W., Everts, H., Kappert, H. J., Frehner, M., Losa, R. \& Beynen, A. C. (2003). "Effects of Dietary Essential Oil Components on Growth Performance, Digestive Enzymes and Lipid Metabolism in Female Broiler Chickens," British Poultry Science, 44 450457.

Lee, K. W., Everts, H., Kappert, H. J., Van Der Kuilen, A. G., Lemmens, M. \& Frehner Beynen, A. C. (2004). "Growth Performance, Intestinal Viscosity, Fat Digestibility and Plasma Cholesterol in Broiler Chickens Fed a Rye-Containing Diet Without or With Essential Oil Components," International Journal of Poultry Science, 3 613-618.

McEvoy, J. D. G. (2002). "Contamination of Animal Feed Stuffs as a Cause of Residues in Food: A Review of Regulatory Aspects, Incidence and Control," Analytica Chimica Acta, 473 3-26.

Oviedo-Rondo'N, E. O., Hume, M. E., Hernandez, C. \& Clemente-Hernandez, S. (2006). "Intestinal Microbial Ecology of Broilers Vaccinated and Challenged with Mixed Eimeria Species, and Supplemented with Essential Oil Blends," Poultry Science, 85 854-860.

Remmal, A., Achahbar, S., Bouddine, L., Chami, N. \& Chami, F. (2011). "In Vitro Destruction of Eimeria Oocysts by Essential Oils," Veterinary Parasitology, 182 121-126.

Remmal, A., Bouchikhi, T., Rhayour, K., Ettayebi, M. \& Tantaoui-Elaraki, A., (1993b). "Improved Method for the Determination of Antimicrobial Activity of Essential Oils in Agar Medium," Journal of Essential Oil Research, 5 1179-84.
Remmal, A., Bouchikhi, T., Tantaoui-Elaraki, A. \& Ettayebi, M. (1993a). "Inhibition of Antibacterial Activity of Essential Oils by Tween 80 and Ethanol in Liquid Medium," Journal de Pharmacie de Belgique, 48 352356.

Rhayour, K., Bouchikhi, T., TantaouiElaraki, A., Sendide, K. \& Remmal, A. (2003). "The Mechanism of Bacterial Action of Oregano and Clove Essential Oils and of Their Phenolic Component on Escherichia Coli and Bacillus Subtillis," Journal of Essential Oil Research, 15 286-292.

Ryley, J. F., Meade, R., Hazelhurst, J. \& Robinson, T. E. (1976). "Methods in Coccidiosis Research: Separation of Oocysts from Faeces," The Journal of Parasitology, 73 311-326.

Schäfer, H., Clauss, W. \& Hörnicke, H. (1984). "Cationophore Properties of the New Polyether Antibiotic Salinomycin Investigated in Distal Rabbit Colon in Vivo and in Vitro," Comparative Biochemistry and Physiology, 79 387-92.

Shirley, M. W. (1995). 'Eimeria Species and Strain of Chickens,' In Eckert, J., Braun, R. Shirley, M.W., Coudert, P. (Eds.), Cost 89/820. Biotechnology. Guidelines on Techniques in Coccidiosis Research.

Stephan, B., Rommel, M., Daugschies, A. \& Haberkorn, A. (1997). "Studies of Resistance to Anticoccidials in Eimeria Field Isolates and Eimeria Strains," Veterinary Parasitology, 69 19-29.

Williams, R. B. (1997). "Laboratory Tests of Phenolic Disinfectants as Oocysticides Against the Chicken Coccidium Eimeria Tenella," Veterinary Record, 141 447- 448.

Wilson, R. G. (1976). "Studies on ShortTerm Application of Drugs to Eimeria Tenella in Tissue Culture," Parasitilogy, 73 283-6. 